\title{
A Vestibular Schwannoma Treated by Radiosurgery Gammaknife: A Case Report
}

Bahalou Mohammed El Habib*, Bouchaib Hemmaoui, Ali Jahidi, Errami Nourreddine, Mohamed Zalagh, Fouad Benariba

Department of Surgery

DOI: $10.36347 /$ sasjs.2020.v06i03.008

| Received: 05.03.2020 | Accepted: 13.03.2020 | Published: 18.03.2020

*Corresponding author: Bahalou Mohammed El Habib

Abstract

Vestibular schwannoma is a benign tumor that develops depending on the schwanne sheath of the vestibular nerve. We report the case of a patient with upper vestibular nerve schwanoma treated by radiosurgery Gamma Knife ${ }^{\circledR}$. This is a 53-year-old patient with no particular history who presents progressive left hearing loss for three years with homolateral tinnitus and recurrent vertigo, without facial paralysis, headache or other neurological symptoms. She has a normal otoscopy with left perception deafness and a positive Halmagy test on the left. The tonal audiometry reveals a left severe hearing loss. A videonystagmography reveals a left deficit syndrome. In the Video-Halmagy impulse test, we have a superior and lateral left semi-circular canals dysfunction. Magnetic resonance imaging reveals a $7 * 5 \mathrm{~mm}$ stage 1 a vestibular schwannoma. The patient received a gammaknife by radiosurgery. The outcomes are favorable. However, the patient had left unilateral cophose and post-therapeutic headaches without facial nerve paralysis. Vestibular schwannoma is a benign tumor who can cause a vital dysfunction by its localisation. Therapeutic management must ensure tumor control without being aggressive.

Keywords: vestibular schwannoma, perception deafness, Gamma Knife®.

Copyright @ 2020: This is an open-access article distributed under the terms of the Creative Commons Attribution license which permits unrestricted use, distribution, and reproduction in any medium for non-commercial use (NonCommercial, or CC-BY-NC) provided the original author and source are credited.

\section{INTRODUCTION}

Vestibular schwannoma, falsely called acoustic neurinoma, is a benign tumor that develops at the schwanne sheath of the vestibular nerve. It causes by its evolution, a cranial nerves dysfunction handicapping the patient with a risk to engage the vital prognosis in advanced stages. It accounts for about $8 \%$ of intracranial tumors and $90 \%$ of ponto-cerebellar tumors. The diagnosis is based on magnetic resonance imaging. Treatment is first and foremost surgical, requiring a complete resection with a preservation of the facial nerve and hearing.
We report a case of a patient with upper vestibular nerve schwanoma treated by radiosurgery gammaknife.

\section{CLINICAL OBSERVATION}

This is a 53-year-old patient with no particular history who presents progressive left hearing loss for three years with homolateral tinnitus and recurrent vertigo, without facial paralysis, headache or other neurological symptoms. She has a normal otoscopy with left perception deafness and a positive Halmagy test on the left. The tonal audiometry reveals a left severe hearing loss. A videonystagmography reveals a left deficit syndrome (Figure 1). 


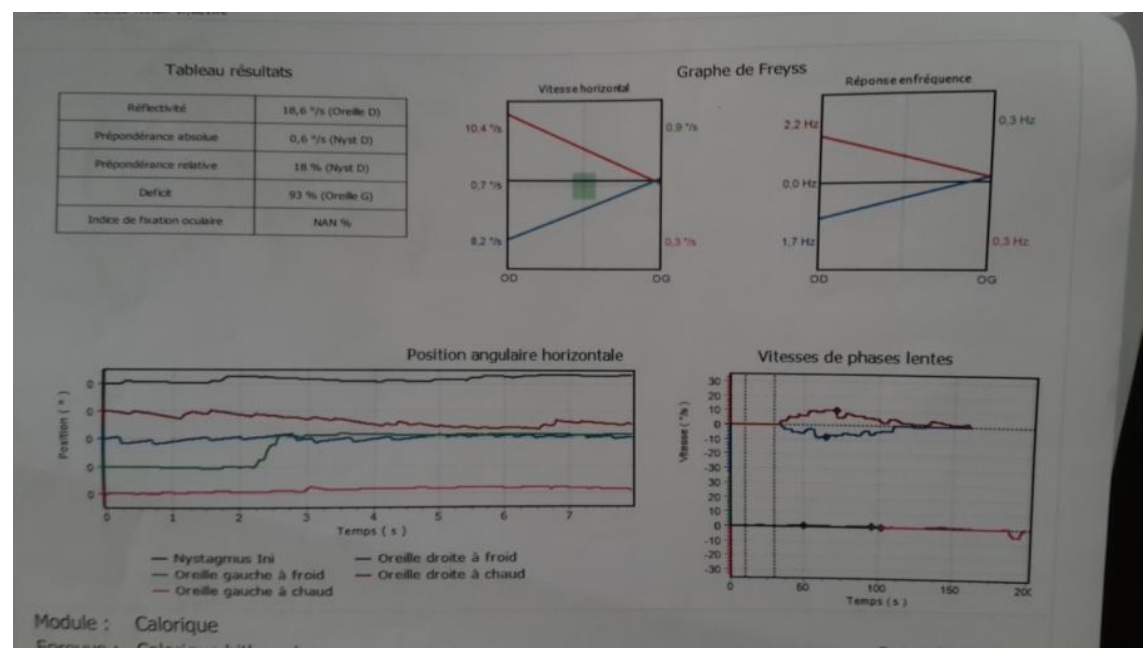

Fig-1: Left deficit canal in caloric test

In the Video-Halmagy impulse test, we have a superior and lateral left semi-circular canals dysfunction (Figure 2). Magnetic resonance imaging reveals a $7 * 5$ $\mathrm{mm}$ stage 1 a vestibular schwannoma. The patient received a gammaknife by radiosurgery. The outcomes are favorable. However, the patient had left unilateral cophose and post-therapeutic headaches without facial nerve paralysis.

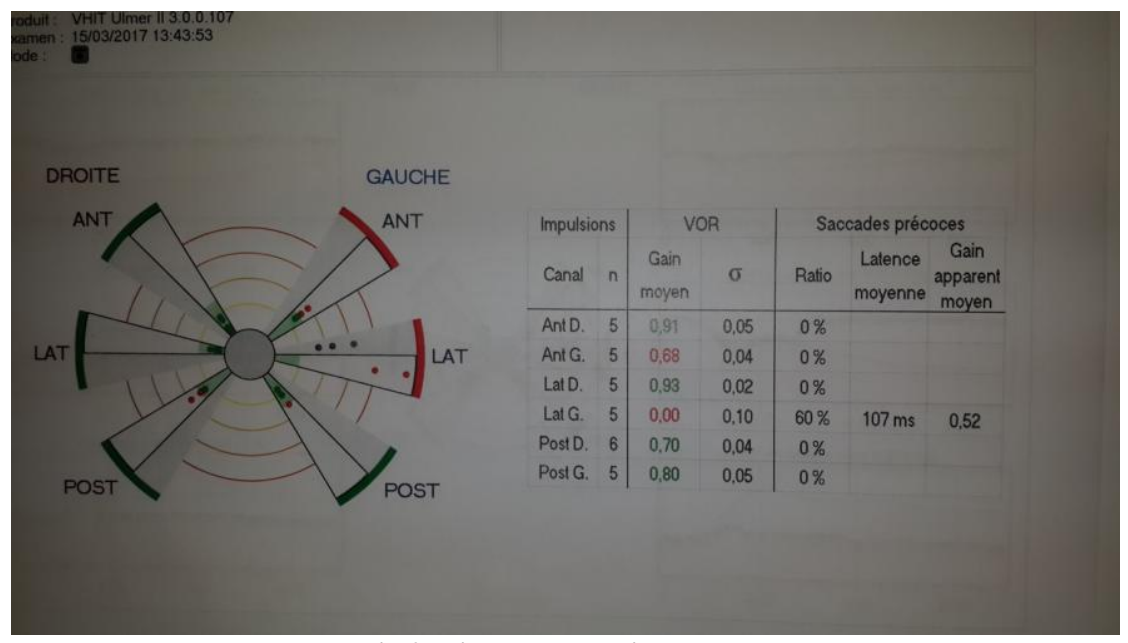

Fig-2: Video Halmagy impulse test

\section{DISCUSSION}

Vestibular schwannoma, falsely called acoustic neurinoma, is a benign tumor that develops at the schwanne sheath of the vestibular nerve, as its name suggests. The First description is published by House and Luetg in 1777 [1].

It accounts for about $8 \%$ of intracranial tumors and $90 \%$ of ponto-cerebellar tumors [2]. Histologically, the tumor contains a mixture of dense, fibrillary Antoni A type tissue and loose Antoni B type tissue. Macroscopically, vestibular schwannoma presents itself as a yellowish formation, classically «buff yellow», often heterogeneous with grey or pinkish areas. It is often rounded, globular and limited by a pseudoarachnoid capsule. In immunohistochemistry, it reacts intensely and homogeneously with PS 100, Leu-7 and vimentine [1]. It occurs in adults in their fifties with a sex ratio close to 1 in favor of a slight female predominance.
Hearing loss is one-sided neurosensory hearing loss and tinnitus is the number one cause. It is especially evident in telephone conversations, where the difference in hearing can be clearly demonstrated. However, sudden installation deafness is not uncommon. Other manifestations are reported to reflect more or less the extension of the tumor process such as facial neuralgias, hypoesthesia of the face, mixed nerve damage or disturbance of balance in the context of cerebellar syndrome. The facial nerve is long preserved [3].

The Metz test, if performed, evokes a retrocochlear origin of deafness. The early auditory potentials evoked have a sensitivity of about $95 \%$. Their normality does not allow eliminating the diagnosis. The signs suggestive of a retrocochlear origin are: the delay of the V-wave, the increase of the interval I-III and I-V, the asymmetry of the interaural interval I-V and the absence of a trace. 
Magnetic resonance imaging is the best diagnostic method. The tumor appears in hyperT2 T1 iso-signal enhancing after gadolinium injection [1].

The treatment is surgical. Radiosurgery is indicated in well-selected patients or refusing surgical treatment. Gamma-Knife Stereotactic Radiosurgery (GK-SRS) is currently considered a valid and approved alternative for small to medium sized tumours. Radiation surgery requires armed surveillance by successive imageries demonstrating the absence of neurinoma evolution [4]. It should be noted that patients treated with radiation surgery took an average of 7 days off at the time of treatment and $99 \%$ returned to work, while those treated surgically were absent from work for 130 days on average and only $66 \%$ returned to work [4].

Tumor control is the main criterion for evaluating oncology treatment requiring long-term follow-up given the slow growth of schwannoma. Thus, the rate of control of tumours varied from 91 to $97 \%$ over 5 years and from 85 to $95 \%$ at 10 years [5]. The second is to obtain the same therapeutic efficacy with the minium of side effects.

In this context, the preservation of functional hearing ranged mainly from $42 \%$ to $53 \%$ over 5 years, and rates at 10 years decreased to $24-44 \%$ [5]. The best long-term 10-year hearing result was found in 45-yearold patients with a $1.5 \mathrm{~cm} 3$ small-volume tumor and treated within 2 years of MRI diagnosis with grade 1 hearing at the time of radiosurgery. Our patient already had a deteriorated hearing at the time of diagnosis.

The preservation of facial nerve function is crucial given the aesthetic damage and quality of life. It is a determining factor in therapeutic management. Gamma-knife radiosurgery preserves the motor function of a normal face in 96.2 to $99 \%$ of patients (Brackmann grade 1) [5]. The current estimate of the risk of facial neuropathy of any grade is less than $1 \%$. The facial is preserved in our case.
Trigeminal nerve damage is detected in 0.6 to $5.8 \%$ of patients after radiation surgery. It is rather the prerogative of large schwannomas [5].

For radiation-induced malignancies, two recent studies found an incidence of $0.2-0.3 \%$ in their treatment cohorts [5]. These values are low and should not influence therapeutic behaviour.

\section{CONCLUSION}

Vestibular schwannoma is a benign tumor who can cause a vital dysfunction by its localisation. Therapeutic management must ensure tumor control without being aggressive.

\section{Conflict of interest}

The authors do not declare any conflict of interest.

\section{REFERENCES}

1. Soudni Soukaina. Le schwannome vestibulaire (à propos de 28 cas). Thèse de médecine. 2016. Bibliothèque faculté de Fès.

2. Matthew L Carlson, Michael J Link, George B Wanna, Colin L Driscoll. Management of Sporadic Vestibular Schwannoma. Otolaryngol Clin N Am; 2015 http://dx.doi.org/10.1016/j.otc.2015.02.003

3. Hossein Mahboubi, Ronald Sahyouni, Omid Moshtaghi1, Kent Tadokoro, Yaser Ghavami, Kasra Ziai, Harrison W. Lin, Hamid R. Djalilian. CyberKnife for Treatment of Vestibular Schwannoma: A Meta-analysis. Otolaryngol Head Neck Surg. 2017 July; 157(1): 7-15.

4. Carlotta Morselli, Boari N, Artico M, Bailo M, Piccioni LO, Giallini I M. de Vincentiis, Mortini P, Mancini P. The emerging role of hearing loss rehabilitation in patients with vestibular schwannoma treated with Gamma Knife radiosurgery: literature review. Neurosurgical Review. https://doi.org/10.1007/s10143-02001257-8

5. Gregory N, Bowden, Ajay Niranjan L. Dade Lunsford Leksell Radiosurgery for Vestibular Schwannomas. Prog Neurol Surg. Basel, Karger. 2019, 34:82-90. 